\title{
Reabilitação estética anterior com uso de laminados cerâmicos: Relato de caso
}

\author{
Esthetic rehabilitation with ceramic laminates: Case report \\ Rehabilitación estética con laminados cerámicos: Reporte de caso
}

Recebido: 15/05/2021 | Revisado: 21/05/2021 | Aceito: 23/05/2021 | Publicado: 09/06/2021

\author{
Amanda Costa Ferro \\ ORCID: https://orcid.org/0000-0003-2623-2152 \\ Universidade Estadual Paulista, Brasil \\ E-mail: a.ferro@unesp.br \\ César Augusto Abreu-Pereira \\ ORCID: https://orcid.org/0000-0003-0665-9060 \\ Universidade Estadual Paulista, Brasil \\ E-mail: cesar.abreu@unesp.br \\ Emerson de Sousa Pinheiro \\ ORCID: https://orcid.org/0000-0003-3844-470X \\ Universidade de Brasília, Brasil \\ E-mail: emersonpisousa@gmail.com \\ Beatriz Ribeiro Ribas \\ ORCID: https://orcid.org/0000-0001-6763-7258 \\ Universidade Estadual Paulista, Brasil \\ E-mail: beatriz.ribas@unesp.br \\ Ana Luiza Gorayb Pereira \\ ORCID: https://orcid.org/0000-0002-4933-4736 \\ Universidade Estadual Paulista, Brasil \\ E-mail: analugorayb@gmail.com \\ Rodolfo Israel Rêgo Sousa \\ ORCID: https://orcid.org/0000-0002-4616-9368 \\ Universidade Federal do Maranhão, Brasil \\ E-mail: rodolforego1989@hotmail.com \\ Rosana Costa Casanovas \\ ORCID: https://orcid.org/0000-0002-6871-3491 \\ Universidade Federal do Maranhão, Brasil \\ E-mail: rosana.c.casanovas@hotmail.com \\ Eduardo Buozi Moffa \\ ORCID: https://orcid.org/0000-0002-0372-6830 \\ E-mail: du_moffa@yahoo.com.br
}

Centro Universitário das Faculdades Associadas de Ensino, Brasil

\begin{abstract}
Resumo
Vários fatores contribuem para alterações nos dentes e consequentemente no sorriso, como escurecimento dental, cárie, envelhecimento, erosão química e diastemas. Com o aprimoramento do tratamento de superfície da cerâmica e da adesão aos substratos, os laminados cerâmicos garantiram espaço definitivo no panorama odontológico, tendo em vista longevidade e resultado final funcional e estético. É nesse cenário que surge o presente estudo, cujo objetivo é apresentar detalhadamente a técnica de fechamento de diastemas com uso de laminados cerâmicos. Paciente do sexo feminino compareceu a Clínica de Odontologia da Universidade Ceuma (São Luís-MA), tendo como queixa principal desconforto estético do seu sorriso. Ao exame clínico foi detectado a presença de diastema que persistiu mesmo após tratamento ortodôntico. O tratamento iniciou a partir do clareamento dentário seguido da gengivoplastia do elemento dental 22 que apresentava desarmonia no zênite gengival. Em seguida foram realizadas as etapas do tratamento reabilitador com laminados cerâmicos até a cimentação. Os laminados cerâmicos representam soluções estéticas e funcionais de alta qualidade para fechamento de diastemas. O sucesso está ligado diretamente com a correta indicação e planejamento, ao preparo suficientemente invasivo, conhecimento das técnicas e materiais, obtendo assim um sorriso estético e harmonioso, com grande garantia de longevidade.
\end{abstract}

Palavras-chave: Diastema; Cerâmicas; Estética dentária.

\begin{abstract}
Several factors contribute to alterations in the teeth and consequently in the smile, such as tooth discoloration, caries, aging, chemical erosion and diastemas. With the improvement of the ceramic surface treatment and the adhesion to the substrates, the ceramic laminate veneers guaranteed a definitive space in the dental practice, in view of longevity, functionality and aesthetic final result. It is in this scenario that the present study appears, which purpose is to present in detail a method of closing diastemas with the use of ceramic laminate veneers. A female patient attended at Ceuma
\end{abstract}


University (São Luís-MA), with the main complaint of aesthetic dissatisfaction of her smile. Clinical examination revealed the presence of diastemas which persisted even after orthodontic treatment. The treatment started with tooth whitening followed by the gingivoplasty of one dental element, which presented disharmony in the gingival zenith. Then, the steps of the rehabilitation treatment with ceramic laminates were carried out until cementation. Ceramic laminate veneers represent high quality aesthetic and functional solutions for closing diastemas. Success is directly linked with the correct indication and planning, with sufficiently invasive preparation, knowledge of techniques and materials, thus obtaining an aesthetic and harmonious smile, with great guarantee of longevity.

Keywords: Diastema; Ceramics; Esthetics dental.

\section{Resumen}

Varios factores contribuyen a cambios en los dientes y consecuentemente en la sonrisa, como oscurecimiento dental, caries, envejecimiento, erosión química y diastemas. Con la mejora del tratamiento superficial cerámico y la adhesión a los sustratos, los laminados cerámicos garantizaron un espacio definitivo en el panorama dental, en vista de la longevidad y resultado final funcional y estético. Es en este escenario donde surge el presente estudio, cuyo objetivo es presentar en detalle la técnica de cierre de diastemas con el uso de laminados cerámicos. Una paciente acudió a la Universidad de Ceuma (São Luís-MA), con la principal queja de insatisfacción estética de su sonrisa. En el examen clínico se detectó la presencia de diastemas que persistieron mismo después del tratamiento de ortodoncia. El tratamiento se inició con un blanqueamiento dental seguido de la gingivoplastia del elemento dental 22 que presentaba desarmonía en el cenit gingival. Luego, se llevaron a cabo los pasos del tratamiento rehabilitador con laminados cerámicos hasta la cementación. Los laminados cerámicos representan soluciones estéticas y funcionales de alta calidad para cerrar diastemas. El éxito está directamente relacionado con la correcta indicación y planificación, con una preparación suficientemente invasiva, conocimiento de técnicas y materiales, obteniendo así una sonrisa estética y armoniosa, con gran garantía de longevidad.

Palabras clave: Diastema; Cerámica; Estética dental.

\section{Introdução}

Vários fatores podem levar a um desequilíbrio estético e funcional dos dentes e, consequentemente, do sorriso, como cárie, erosão, má-oclusão, diastemas e escurecimento dentário (Cardoso et al., 2011). Os diastemas são espaços entre dentes adjacentes na mesma arcada, com etiologia multifatorial, sendo, inclusive, a razão de inúmeras queixas estéticas pelos pacientes. Há diversas técnicas para solucionar o fechamento desses espaços, como restauração direta com resina composta, tratamento ortodôntico e a utilização de restaurações cerâmicas indiretas, como os laminados cerâmicos. A escolha do tratamento ideal deve ser criteriosamente avaliada pelo profissional para que a indicação seja a mais adequada ao caso (Neto et al., 2013).

Os laminados cerâmicos foram apresentados inicialmente por Charles Pincus, em 1938, quando descreveu a técnica em que a porcelana era fixada por um adesivo dentinário durante filmagem de filmes e, logo após, eram removidas, devido à ausência de propriedades adesivas ao substrato dentário. Simonsen e Calamia (1983) e Horn (1983) introduziram conceitos que possibilitaram solucionar a falta de adesividade dos laminados cerâmicos através da adição da etapa de condicionamento ácido das cerâmicas, melhorando substancialmente a fixação das peças protéticas (Peumans et al., 2000).

Os laminados cerâmicos atingem altos índices de sucesso clínico e satisfação do paciente, justamente por serem restaurações duráveis e resistentes quando corretamente indicados (Mazaro et al., 2010). A cerâmica é a principal alternativa de material restaurador, haja vista suas propriedades favoráveis, tais como: resistência à forças de tração, biomimetismo com a estrutura dental, radiopacidade, integridade marginal, estabilidade de cor e outras. A literatura científica atribui o alto desempenho desse tipo de restauração nos âmbitos funcional e estético a essas propriedades, e portanto, maior longevidade das reabilitações estéticas (Amoroso et al., 2012).

Atualmente é possível a confecção de restaurações cerâmicas de espessura reduzida e preparos menos invasivos em razão do avanço das estratégias adesivas das etapas de cimentação. Com isso, os procedimentos indiretos ganharam espaço em tratamentos que antes era realizados de forma direta (Higashi et al., 2012). O uso de laminados cerâmicos é uma solução extremamente estética e é considerado um dos tratamentos mais conservadores para reabilitação estética, com pouca ou nenhuma preparação dentária. Diante disso, aliado ao alto índice de sucesso clínico e grande aceitação dos pacientes, o 
procedimento de correção de alterações dentárias com laminados cerâmicos tem sido cada vez mais utilizado.

Etapas que antecedem a instalação dos laminados cerâmicos para correção da cor do substrato dental, bem como a realização de cirurgias periodontais pode ser necessária para a obtenção de um resultado mais harmônico e natural. Além disso, a realização de um protocolo fotográfico de excelência, aliado ao enceramento diagnóstico e confecção do mock-up auxilia a visualização do caso e a comunicação com o laboratório. Portanto, é importante realizar um planejamento minucioso de todas as etapas do procedimento para a previsibilidade e sucesso final da reabilitação (Venancio et al., 2017).

Desta forma, este trabalho tem como objetivo detalhar o procedimento de reabilitação estética anterior com uso de laminados cerâmicos após clareamento dental e gengivoplastia.

\section{Relato de Caso}

Paciente do sexo feminino compareceu a Clínica de Odontologia da Universidade Ceuma (UNICEUMA), tendo como queixa principal desconforto estético do seu sorriso pela presença de espaços entre os dentes anteriores superiores e tamanho insatisfatório (Figura 1). Inicialmente a paciente foi orientada sobre as questões éticas e de uso de imagem para fins científicos/didáticos e assinou o Termo de Consentimento Livre e Esclarecido (TCLE). No exame clínico intraoral, foi detectado presença de diastema interincisivo, que persistiu mesmo após o tratamento ortodôntico prévio. A paciente possuía uma adequada higiene oral e saúde periodontal e ausência de cáries dentárias.

Figura 1. Fotografia inicial com a vista frontal do sorriso.

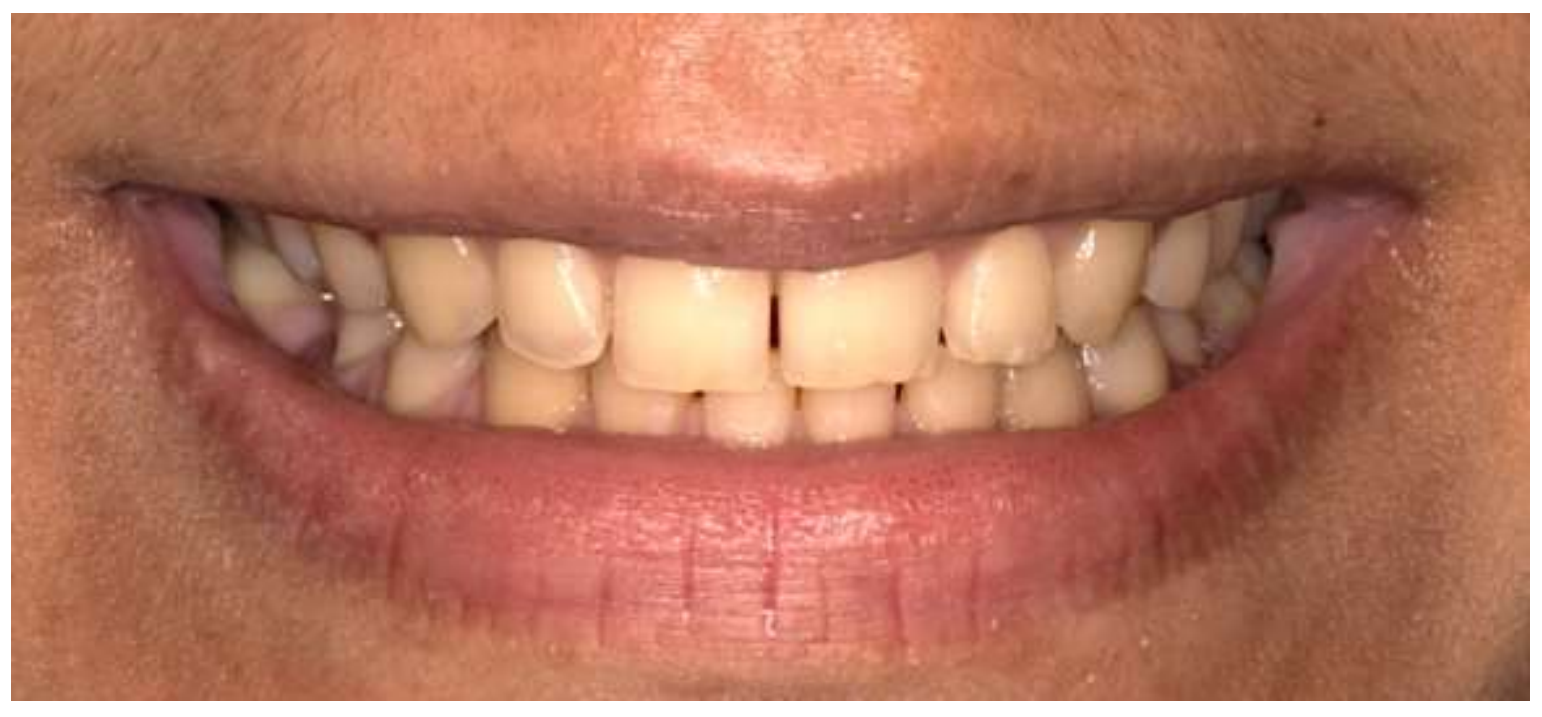

Fonte: Elaboração própria.

Em relação ao plano de tratamento, acordou-se junto com a paciente associar clareamento dentário para melhorar a melhorar a cor do substrato, e em seguida a confecção de laminados cerâmicos. Nesse momento foi planejada a realização de gengivoplastia no elemento dental 22, pois o mesmo apresentava uma diferença na altura gengival com posição errônea do zênite. A paciente foi informada sobre as etapas dos procedimentos, sobre os cuidados que deveria tomar, após isso, foi assinado o Termo de Consentimento para iniciar o tratamento proposto.

Definido o plano de tratamento, realizou-se do protocolo fotográfico, com o objetivo de melhorar a comunicação com o laboratório. Em seguida, procedeu-se com a moldagem dos arcos, utilizando moldeiras de estoque e alginato (Hydrogum 5®; Zhermack®). Após o vazamento do molde e obtenção do modelo de estudo, o técnico de prótese procedeu o enceramento diagnóstico dos 6 elementos dentais (Figura 2). 
Figura 2. Enceramento diagnóstico.

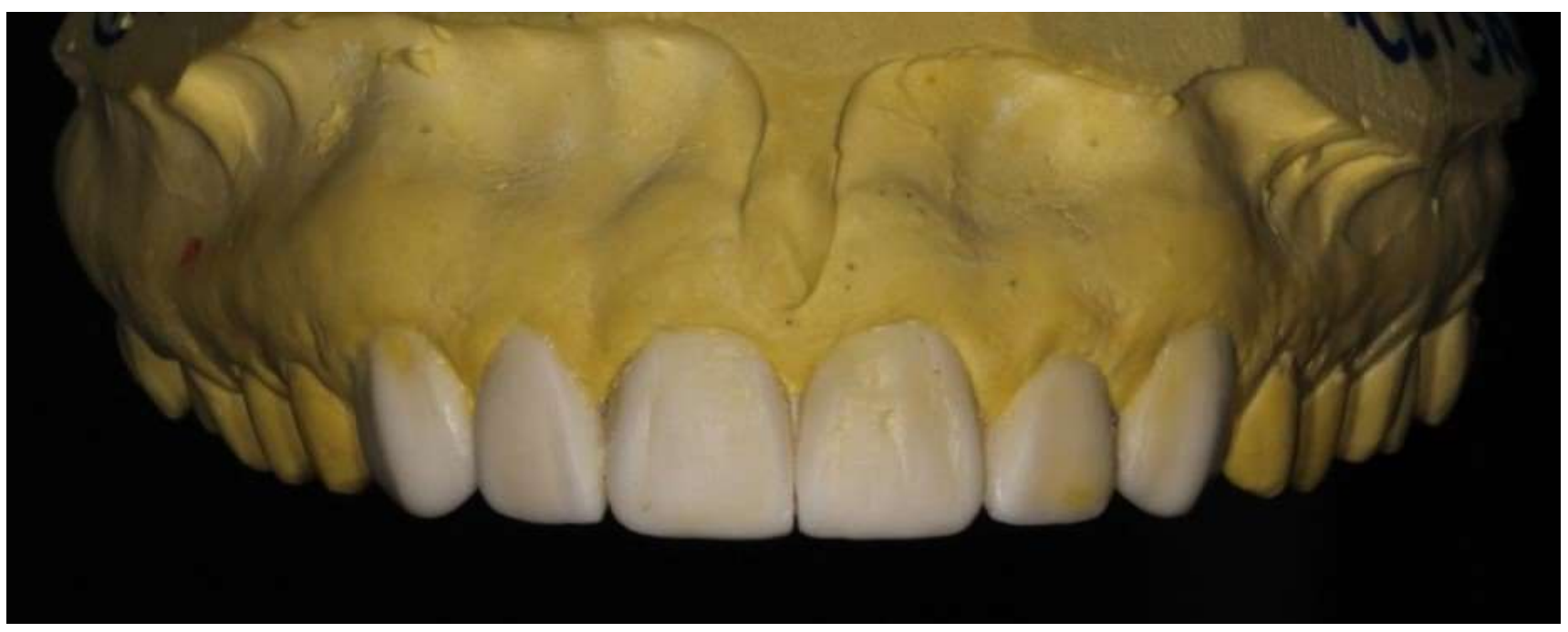

Fonte: Elaboração própria.

Iniciou-se o tratamento com a etapa do clareamento dental, pré-definindo a cor inicial dos dentes A3,5 com escala VITA (Vita Toothguide ${ }^{\circledR}$, Alemanha). Em seguida, procedeu-se o isolamento dos tecidos moles com barreira gengival (Top Dan FGM ${ }^{\circledR}$ Produtos Odontológicos, Joinville, SC, Brasil), recobrindo a gengiva marginal e as papilas, seguida de fotoativação por luz de LED, por 20 segundos para cada grupo de três dentes, formando uma proteção rígida e insolúvel para que não houvesse contato da gengiva com o gel clareador. Foram realizadas duas sessões com gel à base de Peróxido de Hidrogênio a 35\% (Whiteness HP Blue FGM®) manipulado de acordo com as recomendações do fabricante e aplicado sobre a superfície vestibular do esmalte com auxílio de um microaplicador (Cavibrush, FGM® Produtos Odontológicos, Brasil). A cada 5 minutos movimentou-se o gel para que houvesse liberação de eventuais bolhas de oxigênio, renovando dessa forma o contato do gel sobre os dentes. Em cada sessão foram executadas três aplicações de 15 minutos. Ao final do tratamento, o gel foi aspirado com sugador, os dentes foram lavados com água abundante e removida a barreira gengival com ajuda de sonda exploradora. A fim de registrar o resultado final do tratamento, novamente efetuou-se a tomada de cor final com escala VITA após decorrido o tempo necessário para a reidratação dos dentes, sendo registrada a cor A2 imediatamente após a segunda sessão (Figura 3). 
Figura 3. Dentes após a realização do clareamento.

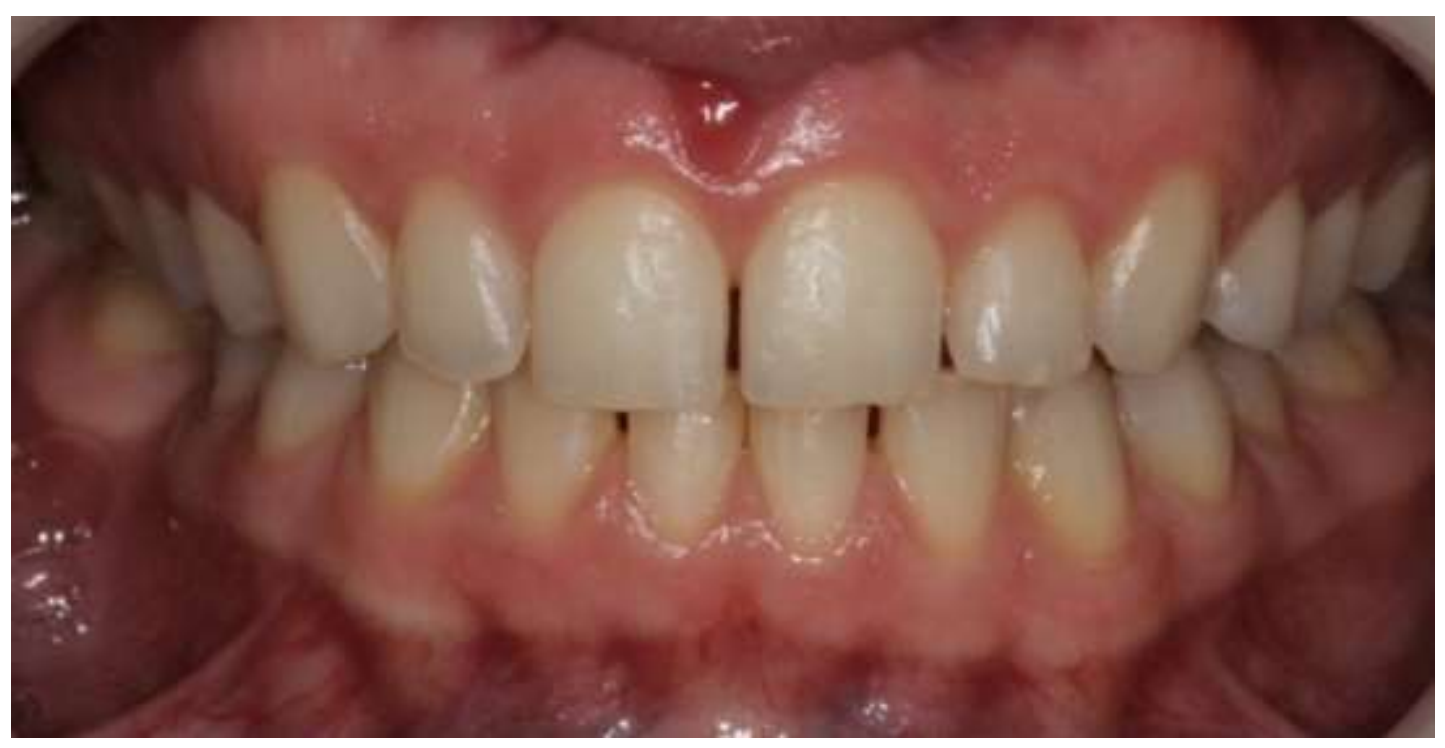

Fonte: Elaboração própria.

Após duas sessões de tratamento clareador, intervalados por 7 dias, procedeu-se a gengivoplastia do elemento 22, com a finalidade de melhorar o zênite e a harmonia estética gengival. Aplicou-se anestesia infiltrativa terminal no nervo alveolar superior anterior e complementação nas papilas. A partir da marcação do zênite no dente 22, foi realizada incisões com bisturi e lamina $15 \mathrm{C}$ para fazer remoção do tecido gengival necessário. Embora o procedimento tenha ocorrido de forma simples e rápida, a paciente foi devidamente orientada aos cuidados pós-operatórios.

Quinze dias após a cirurgia, por meio do enceramento de diagnóstico e das guias de preparo enviadas pelo laboratório, confeccionou-se o mock-up. O molde do enceramento foi recortado com uma lâmina de bisturi $15 \mathrm{C}$ na altura da margem gengival e injetado resina fluida bisacrílica (Structur 2SC, Voco®), cor A1, no interior do mesmo. A guia e o conjunto foram levados à boca até sua completa polimerização, seguido de acabamento e polimento. Esse procedimento possibilitou avaliar se o ensaio restaurador estava adequado com a expectativa estética e as funções de fala, mastigação e oclusão, bem como permitiu uma visualização prévia do tratamento final (Figura 4). Esta etapa é de extrema importância, uma vez que o paciente pode opinar sobre sua satisfação com o tratamento, assim como o profissional tem a liberdade de fazer alterações que julgar conveniente, para estética e função final da reabilitação. 
Figura 4. Realização do mock-up resina bisacrílica.

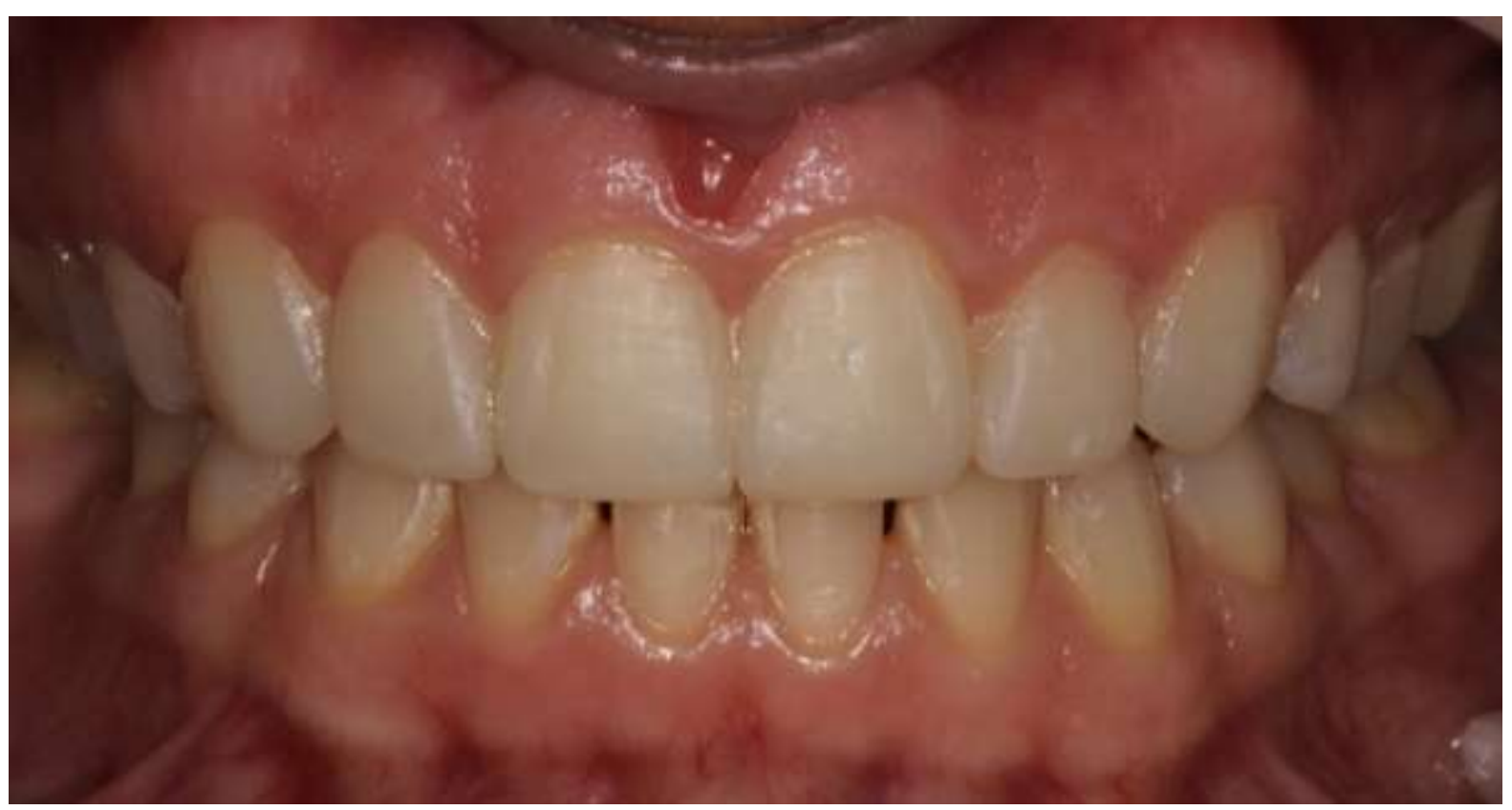

Fonte: Elaboração própria.

O ensaio restaurador foi utilizado como orientação no desgaste no momento do preparo. Foram confeccionadas três canaletas na face vestibular seguindo as inclinações dos terços cervical, médio e incisal, com uso da ponta 834 diamantada (Komet@) (Figura 5a). Na sequência, os preparos foram delimitados com grafite para melhor visualização (Figura 5b). Por meio da marcação de grafite dos guias de preparos verificou-se a necessidade de pequenos ajustes como remoção de ângulos agudos e áreas retentivas. Para isso, foi utilizada a ponta diamantada esférica 1014 (Kg Sorensen®) (Figura 6a) para delimitação do término cervical e a ponta diamantada 3215 (Kg Sorensen®) para realização do bisel na face palatina incisal dos dentes com a finalidade de delimitar o eixo de inserção e adaptação dos laminados. Todos os desgastes dos preparos se restringiram ao esmalte e o acabamento foi feito com discos de lixa (Sof-Lex PopOn®) (Figura 6b).

Figura 5A. Confecção de sulcos de orientação sobre o mock-up com a ponta diamantada 834. Figura 5B. Delimitação dos preparos com grafite.

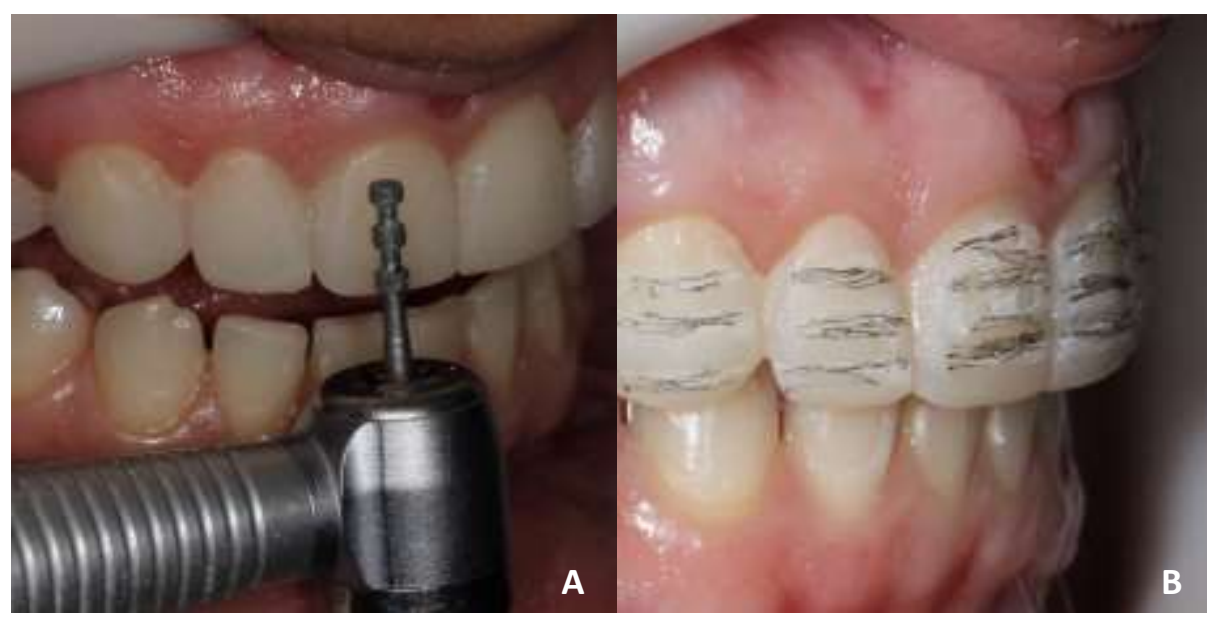

Fonte: Elaboração própria. 
Figura 6A. Preparo cervical com a ponta diamantada 1014. Figura 6B. Acabamento dos preparos com discos de lixa.

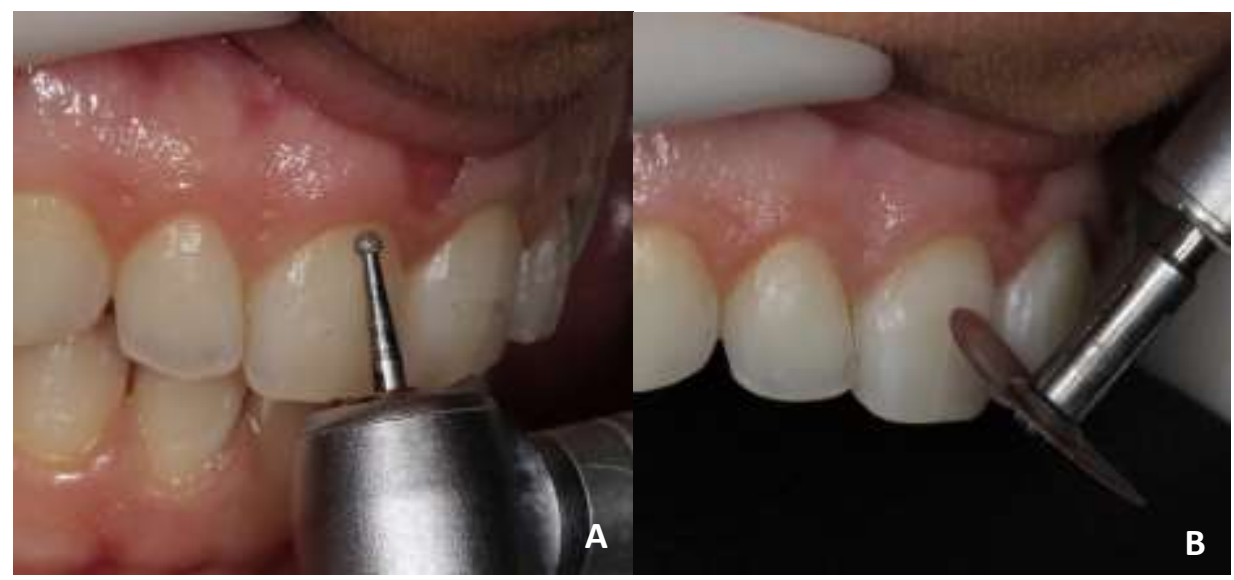

Fonte: Elaboração própria.

$\mathrm{Na}$ mesma sessão, ao final dos preparos, realizou-se a moldagem, utilizando silicone de adição (Elite HD+ Zhermack®). Para melhorar a reprodução do término cervical realizou-se o afastamento gengival, inicialmente inserindo o fio três zeros (000) e dois zeros (00) (Retractor - Roeko®) (Figura 7), respectivamente, no sulco gengival. A moldagem foi realizada através da técnica de dois passos com a moldeira de estoque. Após 5 minutos da inserção do segundo fio afastador, foi realizada a moldagem apenas com material pesado, depois de removido, a pasta leve foi colocada sobre os preparos e sobre o material pesado, desta forma o molde foi reposicionado e aguardada a polimerização final (Figura 8). Terminada a etapa de moldagem, foram confeccionados os provisórios com resina bisacrílica.

Figura 7. Fios retratores $(000$ e 00$)$ inseridos no sulco gengival.

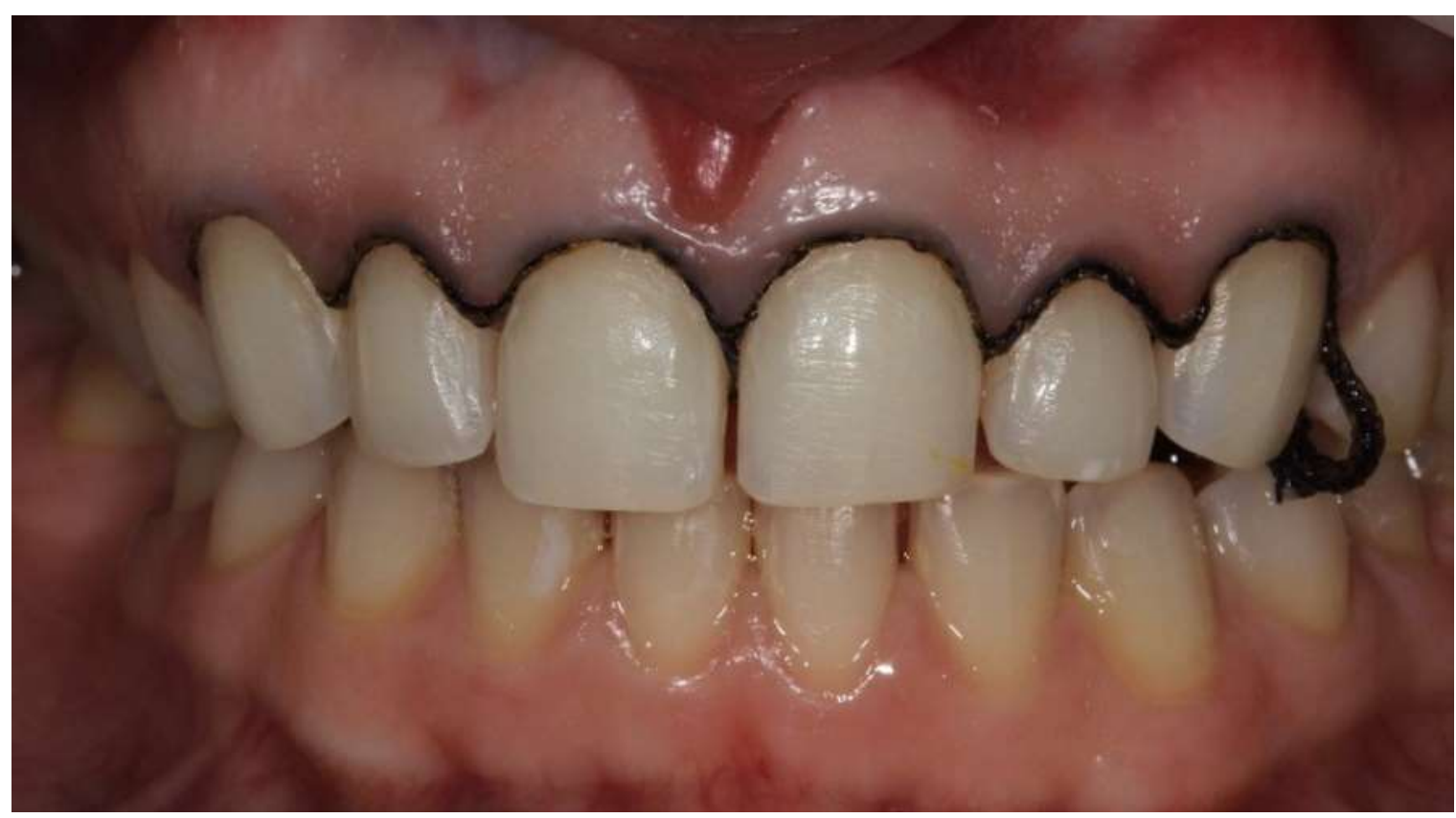

Fonte: Elaboração própria. 
Figura 8. Molde da arcada superior.

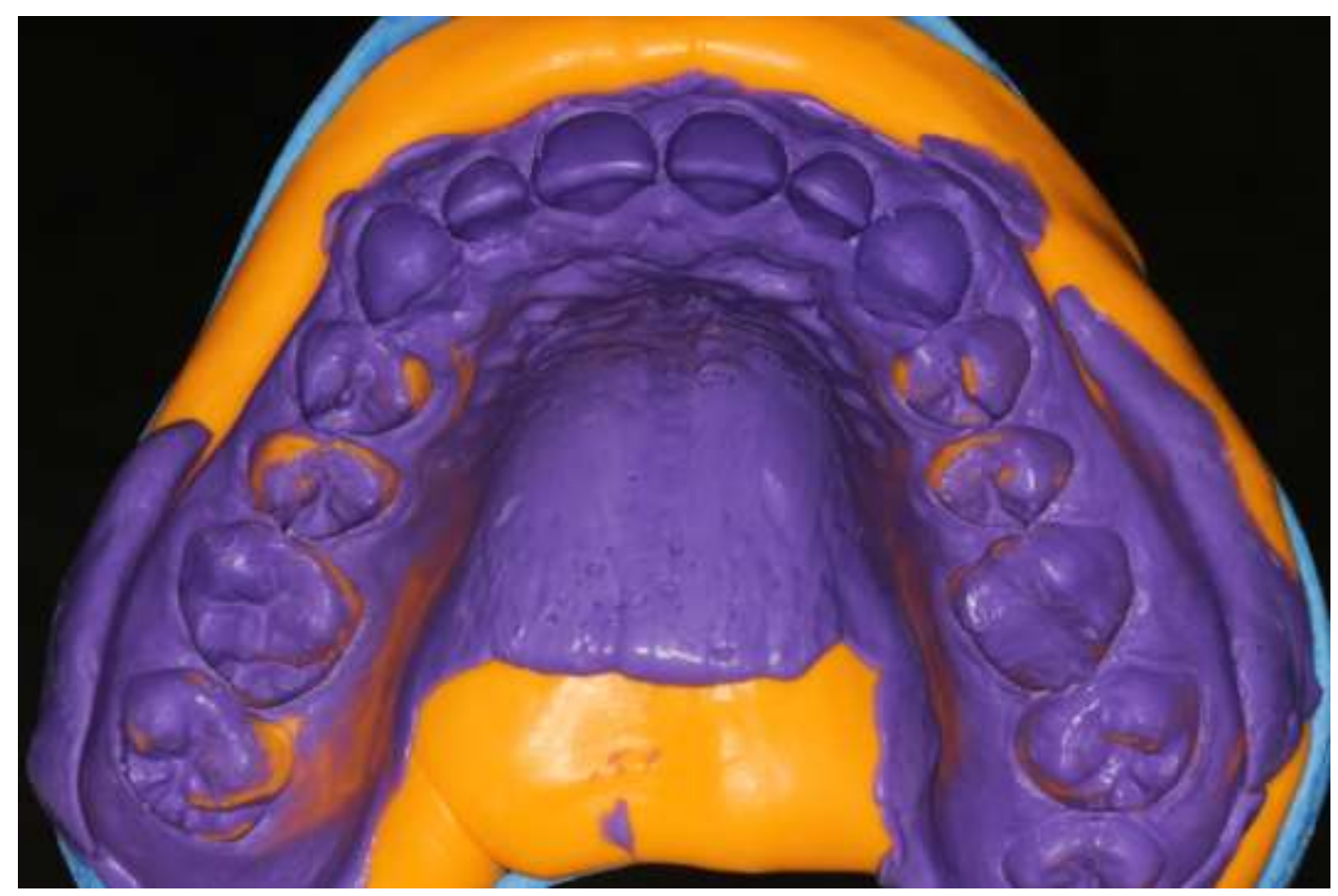

Fonte: Elaboração própria.

O material de escolha para confecção dos laminados cerâmicos foi a cerâmica à base de dissilicato de lítio (IPS E max, IvoclarVivadent $\left.{ }^{\circledR}\right)$, cor A1. Duas semanas depois, a etapa final do tratamento foi iniciada pela prova seca dos laminados cerâmicos, seguida da escolha da cor do cimento resinoso através do teste com as pastas try-in. Para realizar o teste, aplicou-se o produto na parte interna das restaurações cerâmicas e as mesmas foram colocadas em posição. Logo após o teste, selecionouse o cimento de cor transparente (Figura 9).

Figura 9. Prova seca dos laminados cerâmicos.

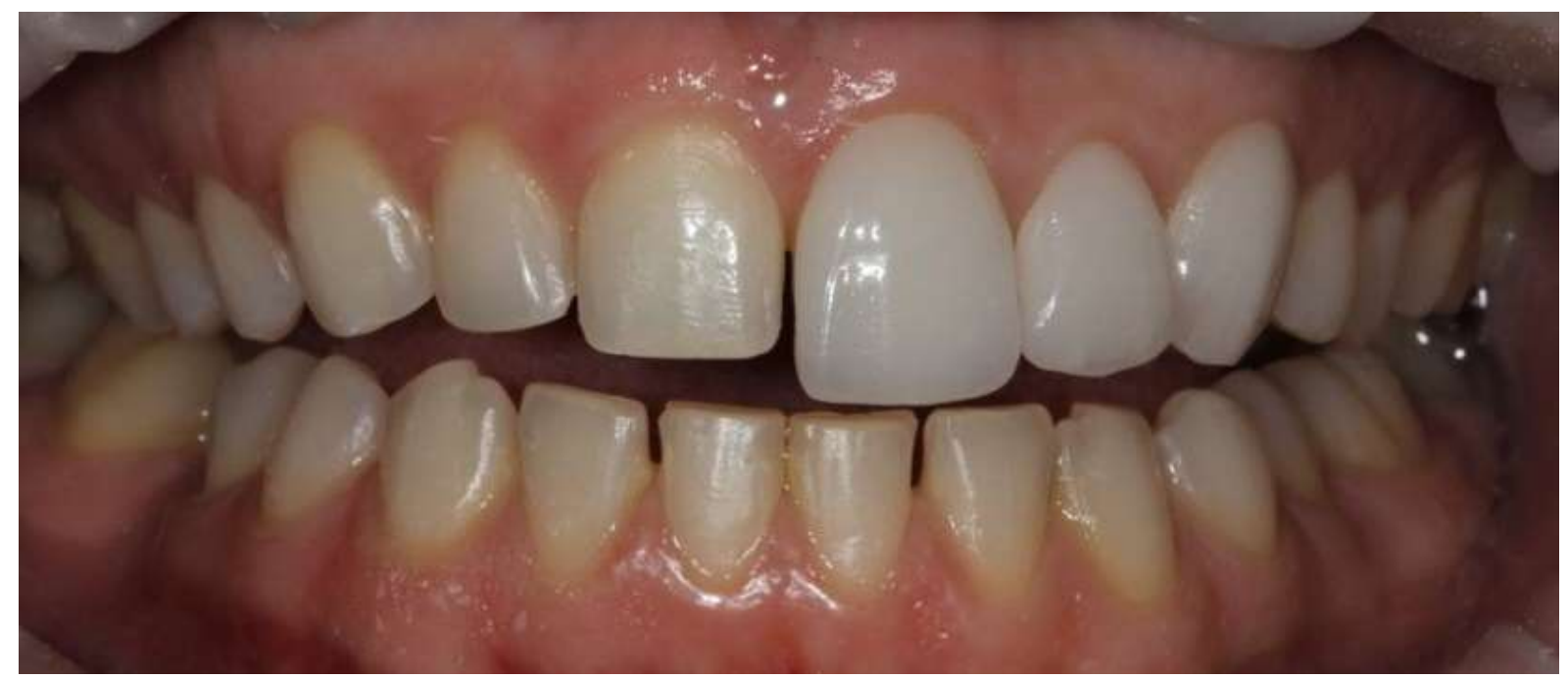

Fonte: Elaboração própria.

Antes de iniciar a etapa de cimentação, foi feita a profilaxia dos elementos dentais com pedra-pomes, água e taça de borracha. O condicionamento ácido do dente foi feito com ácido fosfórico 37\% (N-Etch, Variolink Esthetic LC, 
IvoclarVivadent( $)$, por 30 segundos, seguido de lavagem com jato de ar/água e secagem. Em seguida, aplicou-se o adesivo (Tetric N-Bond Universal, Variolink Esthetic LC, IvoclarVivadent ${ }^{\circledR}$ ), para remover o excesso, utilizou-se um leve jato de ar e, por fim, fotopolimerizado por 20 segundos em cada elemento. O condicionamento ácido dos fragmentos cerâmicos foi realizado com ácido fluorídrico 10\% (Condac Porcelana, FGM®), por 20 segundos, lavagem com jato de ar/água, secagem e aplicação do agente de união (Monobond N, Variolink Esthetic LC, IvoclarVivadent ${ }^{\circledR}$ ), por 60 segundos, acompanhado com jato de água/ar. O cimento resinoso fotopolimerizável (Variolink Esthetic LC, IvoclarVivadent $®$ ) foi inserido em cada peça protética e posicionado sobre os dentes. A remoção de excessos do cimento foi realizada polimerizando por 2 a 3 segundos, para que houvesse uma presa inicial do material, e com auxílio de uma sonda exploradora e um microaplicador do tipo microbrush, os excessos foram removidos e a fotopolimerização final foi realizada por 60 segundos em cada dente (30 segundos na face vestibular e 30 na face palatina). Por fim, os excessos de cimento foram removidos com lamina de bisturi (Figura 10). A paciente foi orientada sobre a correta higienização dos dentes e a necessidade de consultas de retorno para avaliar periodicamente a adaptação e integridade dos laminados cerâmicos (Figura 11).

Figura 10. Laminados cerâmicos cimentados.

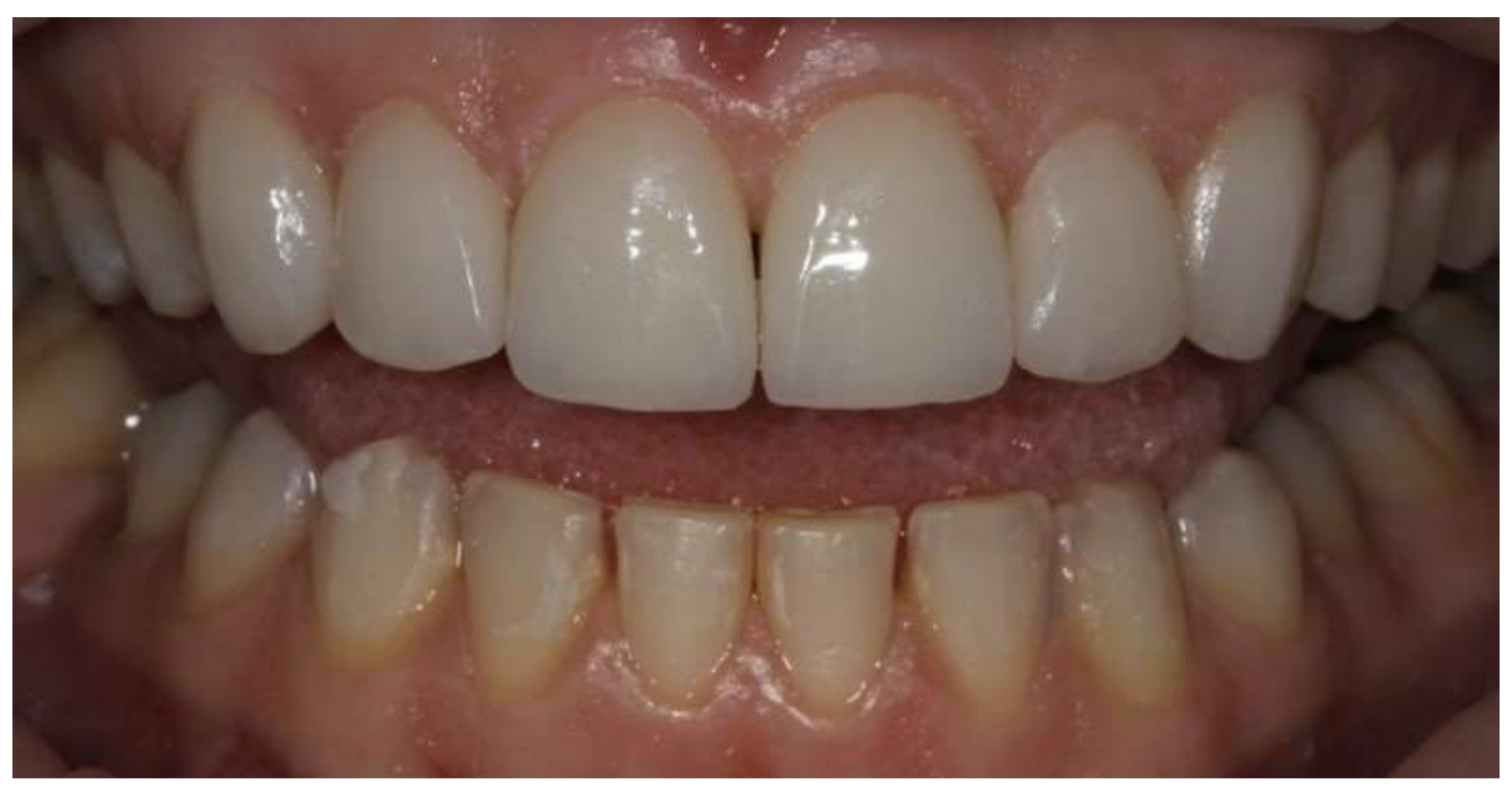

Fonte: Elaboração própria. 
Figura 11. Aspecto do sorriso após a cimentação.

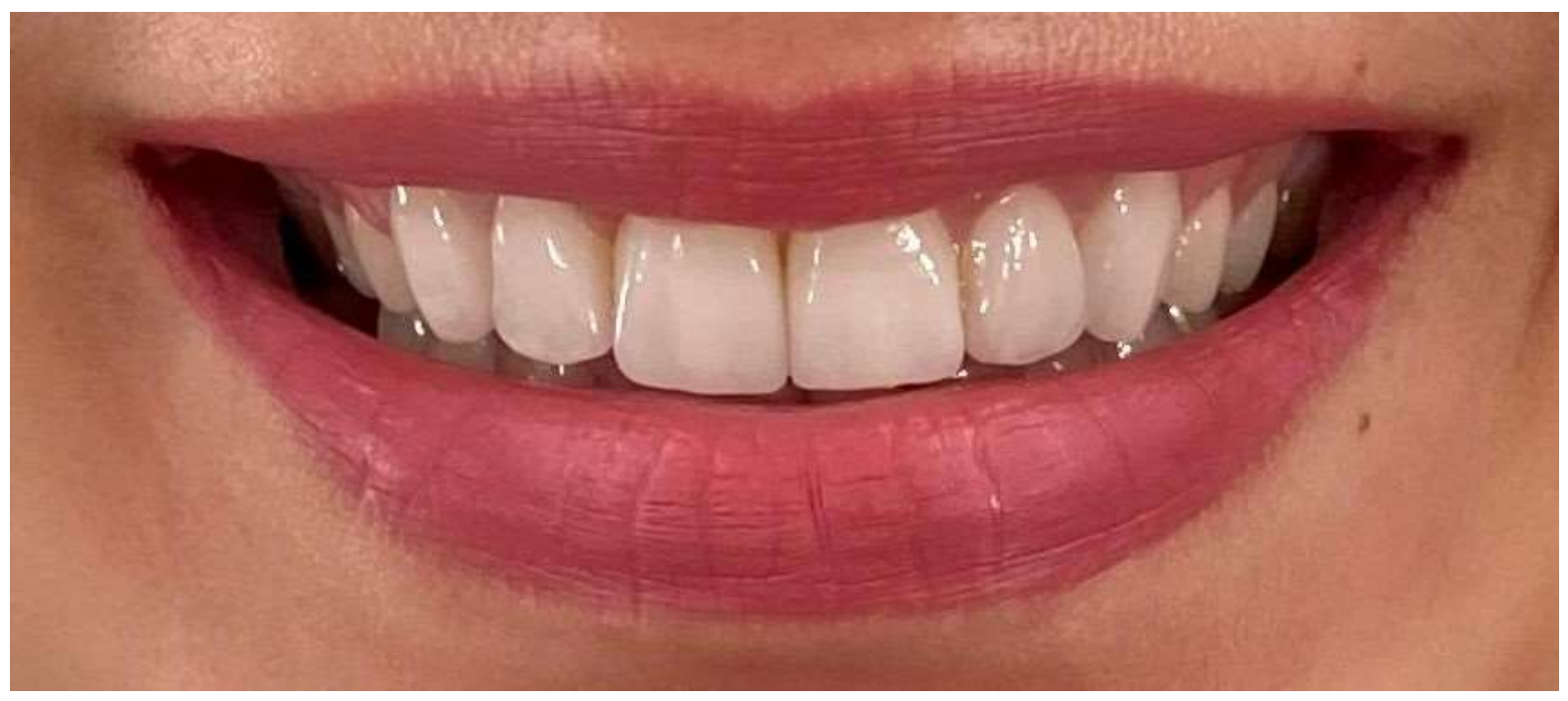

Fonte: Elaboração própria.

\section{Discussão}

Atualmente, a reabilitação oral procurada pelos pacientes não objetiva apenas devolução de forma ou função dos elementos dentários, há a busca também por padrões de beleza, exigindo qualidade estética e sorriso mais harmônico (Soares et al., 2012). Preparos minimamente invasivos para reabilitação com laminados cerâmicos, têm se mostrado uma alternativa de tratamento bem sucedida, tanto na exigência estética quanto na função (Zavanelli et al., 2015).

Outrossim, o adequado diagnóstico e plano de tratamento são fundamentais para o sucesso nos casos de fechamento de diastemas (Cunha et al., 2011). Alterações dentais de forma, cor, posição, tamanho, são frequentes no dia-dia na clínica. Dentre as alterações mais comuns, observa-se com frequência os diastemas ântero-superiores (Araújo et al., 2009), que é fortemente indicado para confecção de laminados cerâmicos (Okida et al., 2016). Ao se comparar restaurações estéticas confeccionadas em resina composta com as de cerâmica, estas possuem vantagens, principalmente relacionada à longevidade, estabilidade de cor, resistência ao desgaste e maior resistência mecânica à fratura, proporcionando um tratamento de sucesso à longo prazo (Soares et al., 2012), fatores estes que foram essenciais para a tomada de decisão clínica diante da possibilidade da utilização dos dois materiais.

Em busca da excelência estética aos dentes anteriores, deve-se fazer uma criteriosa avaliação clínica. Por vezes a reabilitação necessita que procedimentos prévios sejam realizados para que obter uma harmonia estética entre o substrato dental, tecidos periodontais e a face. Diante dessa premissa, a inclusão de uma etapa de plástica gengival para a correção da altura das margens gengivais pode ser necessária, bem como a avaliação da translucidez e opacidade do remanescente dental faz com que seja indispensável o clareamento prévio para alcançar o sucesso final, e sem dúvida contribuir para um desgaste mais conservador (Zavanelli et al, 2015). Ainda que as propriedades estéticas e mecânicas das cerâmicas tenham aumentado consideravelmente, fatores como conhecimento teórico e habilidade do operador devem ser levados em consideração, pois podem influenciar positivamente ou negativamente no resultado final (Souza et al., 2016).

$\mathrm{O}$ uso de laminados cerâmicos no restabelecimento estético e funcional do sorriso está embasado cientificamente por estudos que demonstram seu elevado potencial estético e excelente integração com os tecidos periodontais. Em relação à escolha da técnica restauradora, o uso de facetas cerâmicas possibilita reestabelecimento e manutenção da biomecânica do dente. Isso só é possível em razão dos preparos minimamente invasivos, com mínima remoção de tecido dentário, favorecendo a adesão ao esmalte dentário (Rodrigues et al., 2012). Além disso, o resultado final do tratamento restaurador pode ser obtido 
com pouco ou nenhum preparo no elemento dentário, lançando mão da técnica de preparos guiados, tendo como auxílio o enceramento diagnóstico, guias de preparo e o mock-up (Vaz et al., 2015).

As cerâmicas reforçadas com dissilicato de lítio apresentam em sua matriz vítrea os cristais dispersos de maneira intercalada, dificultando as trincas em seu interior. Esse sistema possui refração de luz, semelhante à estrutura dentária, sem interferência de translucidez, o que leva a caraterísticas ópticas finais satisfatórias. Da mesma forma, o tamanho do cristal favorece o aumento das propriedades mecânicas da restauração (Menezes et al., 2015; Cassiano et al., 2021).

A relação entre o sistema cerâmico e o cimento resinoso, com as necessidades e características de cada situação clínica, deve ser de conhecimento do profissional, para obtenção de um resultado adequado. Dessa forma, torna-se fundamental as provas das restaurações cerâmicas com as pastas try-in no intuito de minimizar possíveis erros e definir resultados com segurança após a cimentação (Cardoso et al., 2011).

O conhecimento da composição do cimento que se utiliza é de fundamental importância. A alteração de cor de cimento resinoso ocorre principalmente devido à oxidação da amina, fator interno na composição química do material, necessário para iniciação da polimerização. Usualmente, em cimentos fotopolimerizáveis, a amina alifática é a de maior quantidade, enquanto que em cimento com polimerização química, a amina terciária aromática é a mais utilizada. De acordo com a literatura, a amina terciária acromática é a que mais sofre com processo de oxidação, logo, os cimentos com polimerização química sofre maior alteração de cor, comparado com os fotopolimerizáveis (Miranda et al., 2009)

Durante o planejamento e análise do tratamento estético, além de necessidades e anseios individuais, deve haver uma boa comunicação profissional e paciente. Esse é o momento oportuno para o profissional sentir as expectativas do paciente acerca do resultado final do tratamento, devendo esclarecer eventuais dúvidas, inclusive, sobre as limitações. As melhorias na aparência geram efeitos psicológicos positivos, beneficiando a autoestima do paciente e, por via reflexa, tornam os procedimentos estéticos conservadores individualmente recompensadores (Louro et al., 2009).

\section{Conclusão}

Com demonstrado pelo resultado do caso descrito neste trabalho, os fragmentos cerâmicos representam soluções de excelência para correção de diastemas. O sucesso do tratamento está relacionado diretamente com a correta indicação, planejamento, preparo suficientemente invasivo e conhecimento das técnicas e materiais, obtendo assim uma reabilitação que cumpre os requisitos estéticos e funcionais e com grandes garantias de longevidade.

\section{Referências}

Amoroso, A. P., Ferreira, M. B., Torcato, L. B., Pellizzer, E. P., Mazaro, J. V. Q., \& Gennari Filho, H. (2012). Cerâmicas odontológicas: propriedades, indicações e considerações clínicas. Revista odontológica de Araçatuba, 19-25.

Bezerra, R. B., Pitta, L. D. A. P., Silva, D. M. D., \& Silva, E. V. F. D. (2014). Reabilitação estética e funcional do sorriso: Relato de caso clínico. Rev. Odontol. Araçatuba (Online), 34-37.

Calamia, J. R., \& Simonsen, R. J. (1983). Tensile bond strength of etched porcelain. J Dent Res, 62.

Cardoso, P. C., Cardoso, L. C., Decurcio, R. A., \& Junior, L. M. (2011). Restabelecimento estético funcional com laminados cerâmicos. Revista Odontológica do Brasil Central, 20(52).

Cassiano, C. K. P., Dias, S. C., Rigolin, F., de Oliveira Mussel, R. L., dos Santos, L. M., \& Tiossi, R. (2021). Shear bond strength between resin cement and lithium disilicate ceramics after intrinsic staining. Research, Society and Development, 10(3).

da Cunha, L. F., Mondelli, J., \& Furuse, A. Y. (2011). Planejamento e considerações pré e pós-operatórias no fechamento de diastemas. Revista Brasileira de Odontologia, 68(1), 12.

de Araujo, É. P., Rocha Filho, L. A., Brum, G. T., \& Caldo-Teixeira, A. S. (2009). Fechamento de diastemas com restaurações diretas de resina composta Relato de Caso Clínico. Revista Gestao \& Saúde, Curitiba, 1(3), 33-38. 
Research, Society and Development, v. 10, n. 6, e54410616141, 2021

(CC BY 4.0) | ISSN 2525-3409 | DOI: http://dx.doi.org/10.33448/rsd-v10i6.16141

de Sousa Menezes, M., Carvalho, E. L. A., Silva, F. P., Reis, G. R., \& Borges, M. G. (2015). Reabilitação estética do sorriso com laminados cerâmicos: Relato de caso clínico. Revista Odontológica do Brasil Central, 24(68).

Higashi, C., Junior, A. S. S., Gomes, G. M., Calixto, A. L., Gomes, O. M. M., \& Gomes, J. C. (2012). Laminados cerâmicos minimamente invasivos. Full dentistry in Science.

Horn, H. R. (1983). Porcelain laminate veneers bond to etched enamel. Dent. North Am., 27, 671-684.

Louro, R. L., Galazi, D. R., \& Moscon, R. M. (2009). Proporção áurea no restabelecimento de um sorriso harmonioso. Revista Brasileira de Pesquisa em Saúde/Brazilian Journal of Health Research.

Miranda, C. B., Carvalho, C. F. D., Barros, J. V. D., \& Silva, S. M. D. A. (2009). Alteração de cor de cimentos resinosos duais ativados com e sem o emprego da luz. Innov. implant. j., biomater. esthet.(Impr.), 25-31.

Neto, S. A., Pereira, M. A., da Cunha, L. F., \& Garcia, P. P. (2013). Tratamentos estéticos conservadores para o fechamento de diastemas: resinas compostas e cerâmicas odontológicas. Revista Dental Press de Estética, 10(4).

Okida, R. C., Vieira, W. S. C., Rahal, V., \& Okida, D. S. D. S. (2016). Lentes de contato: restaurações minimamente invasivas na solução de problemas estéticos. Rev. Odontol. Araçatuba (Online), 53-59.

Peumans, M., Van Meerbeek, B., Lambrechts, P., \& Vanherle, G. (2000). Porcelain veneers: a review of the literature. Journal of dentistry, 28(3), 163-177.

Quinelli Mazaro, J. V., \& Zavanelli, A. C. (2010). Protocolo para tratamento de diastemas com laminados de porcelana: descrição de caso clínico. Revista Dental Press de Estética, 7(4).

Rodrigues, R. B., Veríssimo, C., Pereira, R. D., Queiroz, C. L., Novais, V. R., Soares, C. J., \& Santos-Filho, P. C. F. (2012). Clareamento dentário associado à facetas indiretas em cerâmica: abordagem minimamente invasiva. Revista Odontológica do Brasil Central, 21(59).

Soares, P. V., Zeola, L. F., Pereira, F. A., de Almeida Milito, G., \& Machado, A. C. (2012). Reabilitação estética do sorriso com facetas cerâmicas reforçadas por dissilicato de lítio. Revista Odontológica do Brasil Central, 21(58).

Souza, M. S., Neto, O. I., de Oliveira, R. S., \& de Castro, S. H. D. (2016). Laminados cerâmicos-um relato de caso. Revista Pró-univerSUS, 7(3), 43-46.

Vaz, M. M., Vaz, E. C., Alves, C. B., Lawder, J. C., Lenza, M. A., Souza, J. B., \& Lopes, L. G. (2015). Utilização do ensaio restaurador como guia de desgaste em reabilitação estética com sistema IPS e. max: Caso clínico. Revista Odontológica do Brasil Central, 24(68).

Venâncio, B. D. O. (2017). Reabilitação estética com laminados cerâmicos minimamente invasivos: relato de caso clínico. Trabalho de conclusão de curso (Graduação). Universidade Federal de Uberlandia.

Zavanelli, A. C., Zavanelli, R. A., Mazaro, J. V. Q., Santos, D., \& Fálcon-Antenucci, R. M. (2015). Tratamento cosmético com lentes de contato e laminados cerâmicos. Archives of health investigation, 4(3). 